\title{
Simulating wide-field optical wavefront propagation through single-layer turbulence
}

\author{
S. J. WeddelI*, R. Y. Webb and P. J Bones \\ Department of Electrical and Computer Engineering \\ University of Canterbury \\ New Zealand \\ steve.weddell@canterbury.ac.nz
}

\begin{abstract}
Optical wavefront propagation over a wide field-of-view (FOV) was modeled on empirical data representing a single, dominant layer of atmospheric turbulence. We found the Taylor hypothesis, commonly assumed and used in simulating wavefront propagation, is not appropriate for wide-field application.
\end{abstract}

(C) 2011 Optical Society of America

OCIS codes: $000.0000,999.9999$.

\section{Introduction}

A key requirement in $\mathrm{AO}$ systems is to minimise the effects of anisoplanatism. Various methods have been proposed to effectively compensate the optical path over field angles that exceed the isoplanatic angle [1-3]. However, as the FOV of optical instruments is increased, a point is reached where turbulence passing over a wide aperture can no longer be assumed to remain static. To accurately model wide-field wavefront propagation, an experiment was devised to assess the validity of Taylor's frozen turbulence hypothesis.

We firstly discuss a method to acquire spatiotemporal ensembles from multiple sources, secondly turbulence profile data is used to provide correlation data ensembles. Lastly, these results are analyzed and simulation methods proposed.

Since the following analysis is based on a spatiotemporal model, the Greenwood frequency is used to express the bandwidth specification for an adaptive optics system [4],

$$
f_{G}=2.31 \lambda^{-6 / 5}\left[\sec \beta \int_{\text {Path }} C_{n}^{2}(h) v_{w}(h)^{5 / 3} d h\right]^{3 / 5},
$$

where $\lambda$ is the wavelength, $h$ is the altitude of the turbulent layer, $v_{w}$ is the wind velocity, $C_{n}^{2}$ is the turbulence structure constant, and $\beta$ is the angle of observation from zenith.

According to theory, if $f_{G}$ is exceeded, the captured wavefront perturbations will not evolve as turbulence moves over the aperture. However, given a wide FOV, it is proposed that temporal correlation is unconstrained and high order aberrations evolve due to the extended period required for turbulence to pass over a wider FOV. However, a spatial constraint on the FOV does not appear in Eq. 1.

The Taylor frozen turbulence hypothesis was proposed by G. I. Taylor in 1938 and was a practical attempt to simplify the analysis of turbulence passing over an aperture [5]. Specifically, the hypothesis states that turbulent air moving over the imaging path of an optical instrument, with winds moving the turbulence at velocity $v_{w}$, has insufficient time to change, as viewed through the aperture, i.e., the turbulence is simply displaced. Essentially, the turbulence remains constant for a period, $\tau_{T}$, which is less than the time required for the turbulence to pass over the aperture. As "frozen" turbulence moves over the aperture, it is replaced by new a perturbation field.

The Taylor hypothesis can be expressed as, $v_{s}\left(t_{2}-t_{1}\right)$ and is related to the space-time correlation function of the atmosphere by [6]

$$
\Gamma_{p_{i}}\left(\Delta \mathbf{x}, t_{2}, t_{1}\right)=\left\langle\exp \left[j \psi_{i}\left(\mathbf{x}, t_{1}\right) \exp \left[-j \psi_{i}\left(\mathbf{x}-\Delta \mathbf{x}, t_{2}\right)\right]\right\rangle,\right.
$$

where $\Delta \mathbf{x}$ is the spatial separation between two points in the pupil, and $\psi_{i}$ is the phase perturbation in the $i$ 'th layer.

Validation of Taylor's hypothesis has been conducted by Gendron et. al by calculating the spatiotemporal crosscorrelation function of wavefront slopes using a H-S WFS [8]. This research was motivated by the suggestion that frozen phase slabs could hold potential advantages, in terms of improving AO efficiency. The following study was devised to test the validity of the Taylor hypothesis over a wide field. 


\section{Experimental study}

The effects of turbulence over a wide FOV were measured at the Mt. John University Observatory (MJUO) using a 1-m telescope and modified curvature wavefront sensor [7]. Wavefront aberrations from three Jovian moons, Io, Europa, and Ganymede were recorded over various field angles between 11:33 and 16:12 GMT on 28 July 2009. Calm, clear conditions, with excellent seeing and a light Easterly wind, $V_{\text {wind }}(0) \approx 2 \mathrm{~m} \mathrm{~s}^{-1}$, prevailed. The wind vector was based on an Hour Angle of between $-02: 10: 12$ and $+02: 02: 06$.

Two firewire CCD cameras were used to measure wavefront phase simultaneously from multiple beacons. Since the angular separation of each beacon varied predictably over time, this could be accurately measured. Secondly, time series data were acquired at a rate exceeding the Greenwood frequency, $f_{G}$, and temporal phase differences in these measured data were compensated by time shifting one of the series by a period commensurate with the estimated wind speed and direction. A correlation coefficient matrix comprising Zernike coefficients, $a_{2}, a_{3}, \cdots, a_{20}$, was constructed, providing independent data analysis of each time series by conducting correlation of data ensembles.

The correlation coefficient from two Zernike sets was calculated, each set representing sources widely separated from each other and sampled at a rate exceeding the Greenwood frequency, $f_{G}$. To maintain the coherence of the atmosphere, given an average wind velocity of $21 \mathrm{~m} \mathrm{~s}^{-1}$ [9] and $r_{0}=0.12 \mathrm{~m}$, the Greenwood frequency is $\approx 75 \mathrm{~Hz}$.

To ensure correlation was performed on these same, time-delayed wavefront data, a temporal shift is applied to the leeward data ensemble, w.r.t. to the windward ensemble. Wind velocity profiles specific to the site, MJUO1V and MJUO2V, were used to correlate data from measured wavefront data [9]. According to Mohr [9], a tropospheric layer at an altitude of $11 \mathrm{~km}$ travels at a wind speed of $12 \mathrm{~m} \mathrm{~s}^{-1}$ and $30 \mathrm{~m} \mathrm{~s}^{-1}$, respectively. Based on these parameters, time series data were temporally shifted by $N_{S}$ samples, where

$$
N_{S}=\frac{\Delta T_{m}}{\Delta T},
$$

and where $\Delta T_{m}$ is the period, based on a wind model for the wavefront to be displaced in the metapupil, and $\Delta T$ is the sample period of the recording system.

By employing wind velocity profiles MJUO1V and MJUO2V, and applying a sampling rate $\Delta T=16.67 \mathrm{~ms}$, Table 1 shows the theoretical number of samples required for temporal phase compensation for the leeward Zernike ensemble over an angular separation, $\theta_{\text {Sep }}$.

Table 1. Temporal phase shift parameter summary, calculated for a dominant, single layer of turbulence at a height of $11 \mathrm{~km}$ and velocities, $V_{1}=12 \mathrm{~m} \mathrm{~s}^{-1}$ and $V_{2}=30 \mathrm{~m} \mathrm{~s}^{-1}$.

\begin{tabular}{lcccccccl}
\hline \hline Ensemble & $\begin{array}{c}\text { Frames } \\
\left(10^{3}\right)\end{array}$ & $\begin{array}{c}\theta_{\text {Sep }} \\
(\mu \mathrm{rad})\end{array}$ & $\begin{array}{c}d_{L_{H}} \\
(\mathrm{~m})\end{array}$ & $\begin{array}{c}\Delta T_{1} \\
\left(V_{1}\right)\end{array}$ & $\begin{array}{c}\Delta T_{2} \\
\left(V_{2}\right)\end{array}$ & $\begin{array}{c}N_{1} \\
\left(V_{1}\right)\end{array}$ & $\begin{array}{c}N_{2} \\
\left(V_{2}\right)\end{array}$ & $\begin{array}{l}\text { Source } \\
\text { beacon }^{a}\end{array}$ \\
\hline JUP_2a28h & 5 & 320 & 3.525 & 0.293 & 0.117 & +17 & +07 & I, II \\
JUP_2a28i & 5 & 560 & 6.163 & 0.513 & 0.205 & +30 & +12 & II, III \\
JUP_2a28a & 10 & 730 & 8.034 & 0.669 & 0.268 & -40 & -16 & II, III \\
\hline \hline
\end{tabular}

${ }^{a}$ Io = I; Europa = II; Ganymede $=$ III.

\section{Results}

A correlation analysis was performed on each set from the three ensembles, JUP_2a28a, JUP_2a28h, and JUP_2a28i. Given the conditions discussed previously, the results of this analysis are shown in Fig. 1.

The correlation coefficient $\rho_{\mathbf{a}, \mathbf{a}^{\prime}}(\cdot)$ was calculated from a time series ensemble pair. Each ensemble comprised a set of Zernike coefficients, $\mathbf{a}$ and $\mathbf{a}^{\prime}$, where $\mathbf{a}$ and $\mathbf{a}^{\prime} \in\left\{a_{2}, a_{3}, \cdots, a_{20}\right\}$. Each Zernike ensemble represented 1000 wavefront maps of time-series image data for each moon. The Pearson's correlation coefficient, $\rho_{X Y}$, is given as

$$
\rho_{X Y}=\frac{\operatorname{cov}(X, Y)}{\sigma_{X} \sigma_{Y}},
$$

where $\sigma_{X}$ and $\sigma_{Y}$ are the standard deviations of $X$ and $Y$, and $\operatorname{cov}(X, Y)$ is the covariance of ensembles $X$ and $Y$, and is valid for, $-1 \leq \rho_{X Y} \leq 1$. 


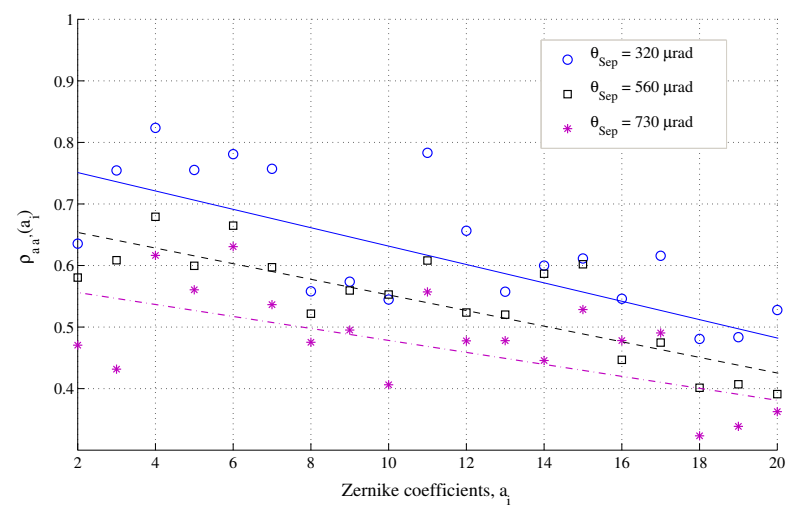

Fig. 1. Correlation of Zernike coefficient data set, $\mathbf{a}=\left\{a_{2}, \cdots, a_{20}\right\}$.

Figure 1 shows distinct groups of three distinct groups of data, where each group represents an ordering of Zernike coefficients, $a_{2}, \cdots, a_{20}$. The group with the highest correlation had the shortest separation, i.e., $320 \mu$ rad. This is followed by the next widest separation, $560 \mu \mathrm{rad}$, and lastly, $730 \mu \mathrm{rad}$. Additionally, Fig. 1 demonstrates how "frozen" turbulence can vary over wide field angles. High-order Zernike coefficients are less correlated than low-order terms; this is expected, since these aberrations contain less energy and momentum, thus susceptible to more chaotic behavior.

\section{Conclusion}

Empirical results from wavefront perturbations of the Jovian moons, used as sources over wide angular separations, demonstrate that turbulence correlation is inversely proportional to field angle. This would suggest that the predictability of turbulence is lost over a wide field and that simplifications, such as application of the Taylor hypothesis, requires careful consideration when applied to simulations. This result, for example, would have consequences for whole-sky correction [10] or spatio-temporal prediction of the point-spread function [11]. Alternative methods, such as evolutionary and dynamic phase screen generation, will be explored.

\section{References}

1. R. Ragazzoni, E. Marchetti, and F. Rigaut, "Modal tomography for adaptive optics," Astron. Astrophys. 342, L53-L56 (1999).

2. T. Fusco, J.-M. Conan, V. Michau, L. M. Mugnier, and G. Rousset, "Efficient phase estimation for large-fieldof-view adaptive optics," Optical Letters 24, 1472-1474 (1999).

3. T. Fusco, J.-M. Conan, L. M. Mugnier, V. Michau, and G. Rousset, "Characterization of adaptive optics point spread function for anisoplanatic imaging. Application to stellar field deconvolution," Astronomy and Astrophysics 142, 149-156 (2000).

4. D. P. Greenwood, "Bandwidth specification for adaptive optics systems," J. Opt. Soc. Am. 67, 390-393 (1977).

5. G. I. Taylor, "Statistical Theory of Turbulence," Royal Society of London Proceedings Series A 151, 421-444 (1935).

6. M. C. Roggemann and B. Welsh, Imaging Through Turbulence (CRC Press, 1996).

7. F. Roddier, "Curvature sensing and compensation: a new concept in adaptive optics," Applied Optics 27, 12231225 (1988).

8. E. Gendron and P. Léna, "Single Layer Atmospheric Turbulence Demonstrated by Adaptive Optics Observations," Astrophysics and Space Science 239, 221-228 (1996).

9. J. L. Mohr, "Atmospheric turbulence characterisation using scintillation detection and ranging," Ph.D. thesis, Dept. of Physics \& Astronomy, University of Canterbury (2009).

10. R. Ragazzoni, E. Marchetti, and G. Valento, "Adaptive-optics corrections available for the whole sky," Nature (UK) 403, $54-6(2000 / 01 / 06)$.

11. S. J. Weddell and R. Y. Webb, "Reservoir computing for prediction of the spatially-variant point spread function," Selected Topics in Signal Processing, IEEE Journal of 2, 624-634 (2008). 